\title{
Mycoplasma pneumoniae associated opsoclonus-myoclonus syndrome in three cases
}

\author{
Benedikt Maria Huber • Susi Strozzi • Maja Steinlin • \\ Christoph Aebi · Simon Fluri
}

Received: 27 May 2009/Accepted: 6 August 2009/Published online: 24 September 2009

(C) Springer-Verlag 2009

\begin{abstract}
Opsoclonus-myoclonus syndrome (OMS) is a rare acquired movement disorder occurring in all age groups, predominantly in infants. Although the exact pathogenesis is still undefined, there is strong evidence for a paraneoplastic or parainfectious immune process resulting in central nervous system dysfunction. Mycoplasma pneumoniae has been implicated in a number of immune-mediated neurologic diseases [28]. However, the association of M. pneumoniae and opsoclonus-myoclonusataxia syndrome is not well established so far. We present three cases with opsoclonus-myoclonus-ataxia syndrome in adolescents following an infection with M. pneumoniae. Monophasic disease course and full recovery correspond to the favorable prognosis known from parainfectious cases in young adults. This should affect therapeutic consideration. OMS should be added to the spectrum of M. pneumoniae-
\end{abstract}

B. M. Huber · S. Strozzi $\cdot$ M. Steinlin $\cdot$ C. Aebi $\cdot$ S. Fluri Department of Pediatrics, University Hospital, Inselspital, Bern, Switzerland

B. M. Huber

Division of General Pediatrics,

Bern, Switzerland

S. Strozzi $\cdot$ M. Steinlin

Division of Pediatric Neurology,

Bern, Switzerland

C. Aebi

Institute for Infectious Diseases,

Bern, Switzerland

\section{S. Fluri $(\bowtie)$}

Department of Pediatrics, Multidisciplinary Neonatal and

Pediatric Intensive Care Unit, University Hospital, Inselspital, CH-3010 Bern, Switzerland

e-mail: simon.fluri@insel.ch associated neurologic complications. Nevertheless, neuroblastoma has to be ruled out in all cases of OMS.

Keywords Opsoclonus-myoclonus-ataxia syndrome . M. pneumoniae $\cdot$ Neurologic disorder P Parainfectious . Paraneoplastic · Immunological mimicry Children . Adolescents

\section{Introduction}

Opsoclonus-myoclonus syndrome (OMS), also known as "dancing eye syndrome", is a rare neurological disorder, characterized by the combination of involuntary, irregular, multidirectional but conjugated eye movements, myoclonus that predominantly involves neck and trunk, and signs of cerebellar ataxia, especially the inability to stand and walk. While the clinical presentation is quite typical, the exact immunopathogenesis, neuroanatomical localization, and pathophysiology are still uncertain [26, 27]. First described in children as "myoclonic encephalopathy of infancy" by Kinsbourne in 1962 [9], it occurs both in children and adults as a paraneoplastic or parainfectious process in association with a variety of neoplastic and infectious diseases. Among the pediatric population, the syndrome is best known between six and 36 months of age and neuroblastomas can be identified in up to $50 \%$ of these cases; in contrast, it is rare in older children and adolescents [21]. In adults, OMS is related to different types of malignant tumors [1,2], but occurs more frequently in association with systemic infection [23]. Although this is known for many different infectious diseases, there is only one recently published case of a four-year-old girl describing the association with Mycoplasma pneumoniae (M. pneumoniae) infection [4]. We present three adolescents with OMS following an infection with M. pneumoniae. 


\section{Case report}

Case 1 A 10-year-old previously healthy girl was sent to our hospital because of uncontrolled eye movements and tremor of the whole body starting 3 days before. History revealed pneumonia 2 weeks earlier. On admission, the girl was afebrile with normal vital signs, but ill appearing and irritable. The neurological examination showed disabling, intermittent, multidirectional, chaotic eye movements and myoclonia of the proximal extremities. No other abnormalities could be found. On the following days, gait impairment and dysmetria occurred as signs of cerebellar ataxia. The girl needed assistance for walking and preferred to keep her eyes closed to reduce oscillopsia. The complete laboratory workup only showed a slight elevation of Creactive protein $(34 \mathrm{mg} / \mathrm{l})$. A lumbar puncture yielded clear, colorless cerebrospinal fluid (CSF) with elevated leukocytes $\left(58 / \mathrm{mm}^{3}\right.$, exclusively mononuclear cell type); glucose and protein concentrations were $5.86 \mathrm{mmol} / \mathrm{l}$ and $0.92 \mathrm{~g} / \mathrm{l}$, respectively. The neopterin level was markedly increased (77.5 nmol/1); other biogenic amines, folates, and pterins were within the normal range. Further CSF investigation demonstrated intrathecal antibody production as well as elevated protein and albumin levels. There was no serological evidence for acute or previous infection with Epstein-Barr virus (EBV) or Borrelia burgdorferi, and CSF analysis was negative for bacterial and enteroviral infection. However, M. pneumoniae-polymerase chain reaction (PCR) was positive in nasopharyngeal secretions and $\operatorname{IgM}$ antibodies were elevated (Table 1). Cranial magnetic resonance imaging (MRI) did not reveal pathologic alterations in the neurocranium, in particular, there were no signs of cerebellar or brain stem inflammation. With normal urine catecholamines, normal abdominal sonography, and chest $\mathrm{X}$-ray, there was no evidence for a paraneoplastic etiology of OMS.

On day 4, the girl was started on methylprednisolone at a dosage of $3.2 \mathrm{mg} / \mathrm{kg} / \mathrm{day}$. The symptoms of OMS slowly improved, but she developed psychotic adverse effects of high-dose steroid therapy. Therefore, the methylprednisolone dosage was reduced and additional therapy with intravenous immunoglobulins was started. The girl was discharged home after 18 days with a persistent slight opsoclonus, myoclonus, and atactic disorder. One month later, apart from a discrete intention tremor, she showed a full recovery without any overt cognitive impairment. The therapy with oral prednisolone was weaned over 10 months. No relapses occurred for a total follow-up of 30 months.

Case 2 A 14-year-old previously healthy girl was referred to our hospital because of a strange movement disorder of her eyes accompanied by unsteadiness, dizziness, nausea, and reduced general condition. Four weeks before admission she suffered from a febrile respiratory disease (sore throat, severe cough). Abnormal findings in the physical examination were diffuse action tremor, myoclonias of the trunk, neck and proximal extremities, signs of cerebellar ataxia with astasia and abasia, and the characteristic finding of opsoclonus. Laboratory tests were normal except for a slight mononuclear pleocytosis $\left(15 / \mathrm{mm}^{3}\right)$, an increased protein level $(0.51 \mathrm{~g} / \mathrm{l})$ as well as positive oligoclonal bands in the clear CSF. CSF level for 5-hydroxyindoleacetic acid was decreased ( $54 \mathrm{nmol} / \mathrm{l})$; level of homovanillic acid was in the lower reference range $(168 \mathrm{nmol} / \mathrm{l})$. Microbiologic investigations could exclude acute infection by EBV, adenovirus, enterovirus, tick-borne encephalitis virus, Borrelia burgdorferi, and group A streptococci. However, complement binding antibody test for $M$. pneumoniae was strongly positive with 1:1,920; IgM antibodies were elevated (Table 1). There was no elevation of catecholamines and their metabolites in a 24-hour urine sample and chest X-ray, sonography of the abdomen as well as cranial MRI were normal.

After an initial deterioration of neurological signs and symptoms, there was marked improvement following therapy with high-dose methylprednisolone $(8.5 \mathrm{mg} / \mathrm{kg} / \mathrm{day})$. The girl was discharged on day 10 with a discreet residual opsoclonus and unsteadiness. Treatment with oral prednisolone was continued and weaned over 4 months. While developing slight and transient signs of hypercortisolism, recovery from OMS was achieved after 4 weeks. A slight hand tremor disappeared during the following months, no relapses occurred during 15 months follow-up.

Case 3 A 14-year-old boy with an uneventful past medical history presented with gait impairment, dizziness, and involuntary chaotic eye movements. He suffered from a febrile infection with sore throat and cough 2 weeks earlier. On admission, he was afebrile and the physical examination revealed opsoclonus accompanied by a truncal ataxia making standing and walking difficult. No other clinical abnormalities and no pathologic results in the standard laboratory workup could be found. The clear and colorless CSF showed a minimal mononuclear pleocytosis $\left(5 / \mathrm{mm}^{3}\right)$, positive oligoclonal bands, but normal glucose and protein levels. CSF level of neopterin was clearly increased (46.6 nmol/1); the level of 5-hydroxyindoleacetic acid was decreased (56 nmol/1). Results of urine catecholamines were normal. In microbiological investigations, the complement binding antibody test for $M$. pneumoniae was strongly positive with 1; 1,280; IgM serology was positive (Table 1). Acute infection with EBV, enterovirus, B. burgdorferi, and group A streptococci was ruled out. Abdominal sonography and cranial MRI were normal, while chest X-ray showed an atypical pneumonia. 


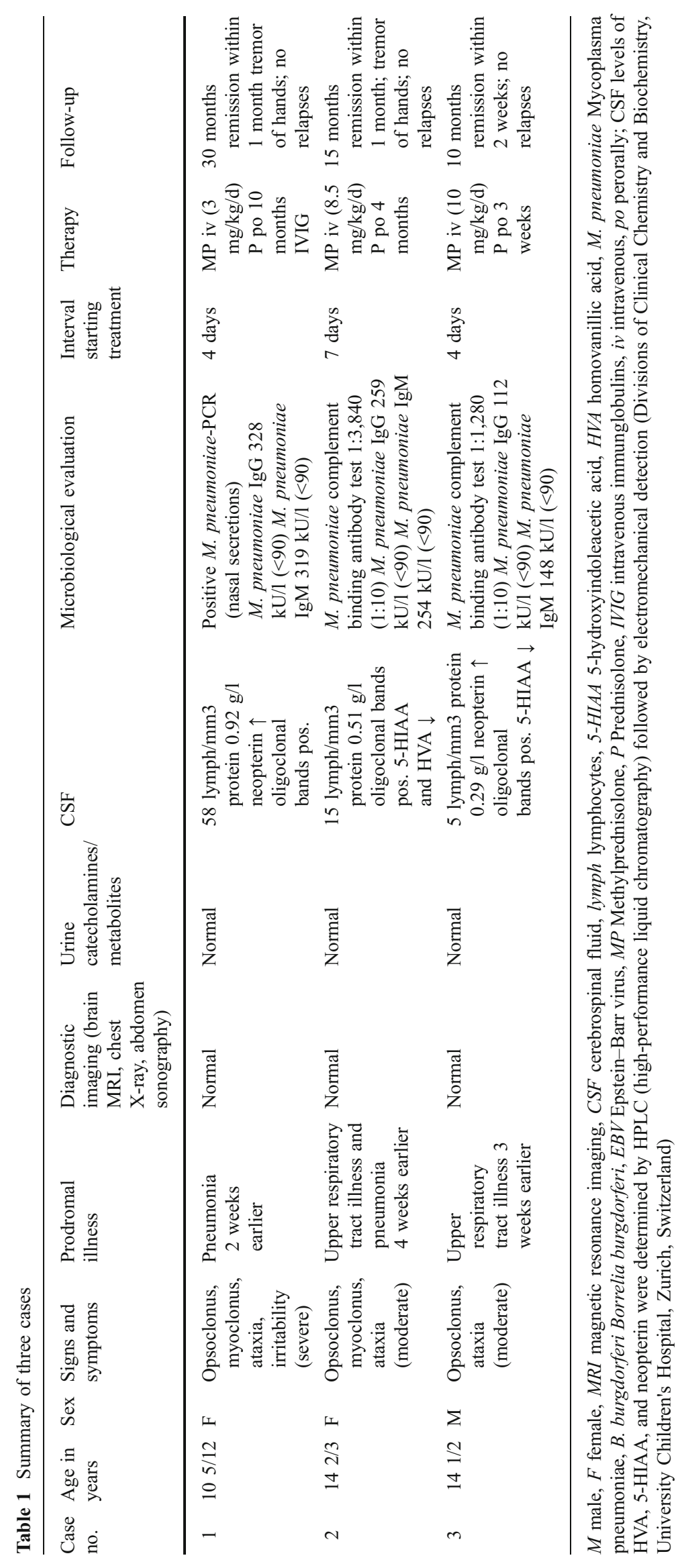


A slight clinical improvement was already observed one day before starting treatment. Under methylprednisolone at an initial dosage of $10 \mathrm{mg} / \mathrm{kg} /$ day, complete remission without residual cognitive or behavioral impairment was achieved within 2 weeks. Therapy with oral prednisolone was weaned over 3 weeks, no relapses occurred for a follow-up of 10 months.

\section{Discussion}

Opsoclonus-myoclonus-ataxia syndrome is a rare acquired movement disorder affecting both children and adults. Although the exact etiology as well as the detailed mechanism of brain injury is still undefined, there is a broad spectrum of evidence for an immune-mediated process, suggesting immune system dysregulation of both humoral and cellular immunity in the context of neoplastic or infectious diseases $[3,5,11,17-20]$. The positive effect of immunomodulatory agents such as corticosteroids or intravenous immunoglobulin, also used in our cases, gives further support to the immunopathogenesis of OMS.

CSF in patients with OMS shows pleocytosis with activated $\mathrm{T}$ and $\mathrm{B}$ lymphocytes in the CSF [18]. B cell overrepresentation leads to intrathecal antibody production. In children with neuroblastoma-related OMS, B cell expansion is thought to be a biomarker of disease activity. Therefore, rituximab, a chimeric monoclonal antibody directed against the CD20 antigen on the surface of mature $\mathrm{B}$ cells, has been successfully used as adjunctive therapy for OMS [16].

In our three cases, no tumor was detected. However, there was strong evidence for a previous or ongoing infection with M. pneumoniae with clearly positive complementbinding antibody test and markedly elevated IgM serology. In the first case, a positive PCR of nasopharyngeal secretions was found. The diagnosis of $M$. pneumoniae infection remains a challenge [25]. The complementbinding antibody test was considered as the standard test for a long time [6]. Because the complement binding antibody test lacks of sensitivity and specificity, the measurement of IgM antibodies with the enzyme-linked Immunosorbent assay is nowadays successfully used for diagnosis of $M$. pneumoniae infection in pediatric patients [24]. To enhance the sensitivity of testing, PCR analysis is sometimes added and should also be done in the CSF if the patient shows neurological symptoms.

This common cause of upper and lower respiratory tract infections are known to be associated with a wide spectrum of extrapulmonary manifestations [12, 24]. Besides direct invasion of affected tissues by M. pneumoniae, immunologic mechanisms are thought to be responsible for the pathogenesis of those disorders. Neurologic manifestations are the most common extrapulmonary complications of M. pneumoniae infections with an overall incidence of about $0.1 \%[8,22]$. The use of a macrolide in patients with a neurologic manifestation consecutive to $M$. pneumoniae infection seems to be reasonable even in the absence of direct central nervous system infection, since macrolides may have direct anti-inflammatory and immunomodulatory properties in addition to their antimicrobial activity [27].

Except for a recently published pediatric case report [4], OMS is not currently listed among the different central nervous system disorders associated with $M$. pneumoniae $[7,12,15]$. The variable CSF abnormalities in M. pneumoniae-associated neurological diseases are frequently characterized by an increased white blood cell count (pleocytosis with lymphocytes predominating), increased protein, and normal to low glucose level [12]. These findings correspond with the results of our cases, and there was additional evidence for intrathecal antibody production as well as an increased neopterin level, which is known as an inflammatory marker indicating $\mathrm{T}$ cell activation and cell-mediated immunity [15]. How the autoimmune reaction contributes to the neurologic disorder in OMS remains unclear, but changes in neurotransmission might play a role. Our findings of decreased CSF levels of the serotonin metabolite 5-hydroxyindoleacetic acid and the dopamine metabolite homovanillic acid as signs of impaired neurotransmission correspond with previously reported data [14].

In infants, where OMS is frequently a feature of neuroblastoma, the course is characterized by multiple relapses and significant developmental sequelae [10, 13]. Consequently, nearly all children require vigorous and prolonged immunosuppressive treatment to control neurologic manifestations [15]. Our cases of OMS in adolescents demonstrate a rather favorable prognosis with a monophasic course and full recovery without neurologic or cognitive deficits. This is comparable to young adults with parainfectious OMS and good response to immunotherapy $[2,27]$.

Therapeutic differences in duration and dosage of corticosteroid administration in our cases result from the initial lack of experience in treating adolescents with OMS and the variable intensity of disease (severe in case 1, moderate in case 2 and 3, respectively). In all three cases, treatment started in the first week of disease manifestation. This contrasts to the long delay in diagnosis and initiation of treatment reported by Tate et al. [21] and may have contributed to the rapid and effective recovery. The clinical improvement even before treatment observed in case 3 could indicate the possibility of spontaneous regression of signs and symptoms in this age group. As a consequence, one can speculate that this subgroup of OMS in adolescence allows a less intense therapeutic procedure without risking multiple relapses and neurodevelopmental sequelae. 
With this knowledge, adverse effects of immunomodulatory treatment, especially of prolonged high-dose corticosteroid administration, could be avoided or at least reduced.

\section{Conclusion}

M. pneumoniae is a frequent cause of respiratory tract infections, and extrapulmonary manifestations are a known phenomenon. OMS seems to be a previously undescribed central nervous system complication of $M$. pneumoniae infection. Compared to paraneoplastic forms of OMS in infants, M. pneumoniae associated cases of OMS in adolescents show a rather benign course without neurodevelopmental long-term sequelae. This affects therapeutic considerations. OMS should be added to the spectrum of $M$. pneumoniae-associated neurologic complications. Nevertheless, neuroblastoma has to be ruled out in all pediatric cases of OMS.

Conflict of interest No conflict of interest has to be reported.

\section{References}

1. Al-Lozi MT, Pestronk A (2001) Paraneoplastic neurologic syndromes. In: Braunwald E, Fauci AS, Kasper DL, Hauser SL, Longo DL, Jameson JL (eds) Harrison's principles of internal medicine, 15th edn. McGraw-Hill, NewYork, pp 636-642

2. Bataller L, Graus F, Saiz A, Vichez JJ (2001) Spanish Opsoclonus-Myoclonus Study Group. Clinical outcome in adult onset idiopathic or paraneoplastic opsoclonus-myoclonus. Brain 124:437-443

3. Bataller L, Rosenfeld MR, Graus F, Vilchez JJ, Cheung NK, Dalmau J (2003) Autoantigen diversity in the opsoclonusmyoclonus syndrome. Ann Neurol 53:347-353

4. Chemli J, Ketata S, Dalhoumi A, Ajmi H, Hassayoun S, Fodha I et al (2007) Opsoclonus-myoclonus syndrome associated with Mycoplasma pneumoniae infection. Arch Pediatr 14:1003-1006

5. Connolly AM, Pestronk A, Mehta S, Pranzatelli MR, Noetzel MJ (1997) Serum autoantibodies in childhood opsoclonus-myoclonus syndrome: an analysis of antigenic targets in neural tissues. $\mathrm{J}$ Pediatr 130:878-884

6. Daxboeck F, Krause R, Wenisch C (2003) Laboratory diagnosis of Mycoplasma pneumoniae infection. Clin Microbiol Infect 9:263-273

7. Daxboeck F (2006) Mycoplasma pneumoniae central nervous system infections. Curr Opin Neurol 19:374-378

8. Guleria R, Nisar N, Chawla TC, Biswas NR (2005) Mycoplasma pneumoniae and central nervous system complications: a review. $\mathrm{J}$ Lab Clin Med 146:55-63

9. Kinsbourne M (1962) Myoclonic encephalopathy of infants. J Neurol Neurosurg Psychiatry 25:271-276
10. Klein A, Schmitt B, Boltshauser E (2007) Long-term outcome of ten children with opsoclonus-myoclonus syndrome. Eur J Pediatr $166: 359-363$

11. Korfei M, Fühlhuber V, Schmidt-Wöll T, Kaps M, Preissner KT, Blaes F (2005) Functional characterisation of autoantibodies from patients with pediatric opsoclonus-myoclonus syndrome. J Neuroimmunol 170:150-157

12. McCormack WM (2001) Mycoplasma infections (2001). In: Braunwald E, Fauci AS, Kasper DL, Hauser SL, Longo DL, Jameson JL (eds) Harrison's principles of internal medicine, 15th edn. McGraw-Hill, NewYork, pp 1073-1075

13. Mitchell WG, Brumm VL, Azen CG, Patterson KE, Aller SK, Rodriguez J (2005) Longitudinal neurodevelopmental evaluation of children with opsoclonus-ataxia. Pediatrics 116:901-907

14. Pranzatelli MR, Huang Y, Tate E, Stanley M, Noetzel MJ, Gospe SM Jr et al (1995) Cerebrospinal fluid 5-hydroxyindoleacetic acid and homovanillic acid in the pediatric opsoclonus-myoclonus syndrome. Ann Neurol 37:189-197

15. Pranzatelli MR, Hyland K, Tate ED, Arnold LA, Allison TJ, Soori GS (2004) Evidence of cellular immue activation in children with opsoclonus-myoclonus: cerebrospinal fluid neopterin. J Child Neurol 19:919-924

16. Pranzatelli MR, Tate ED, Travelstead AL, Barbosa J, Bergamini RA, Civitello L et al (2006) Rituximab (anti-CD20) adjunctive therapy for opsoclonus-myoclonus syndrome. J Pediatr Hematol Oncol 28:585-593

17. Pranzatelli MR, Travelstead AL, Tate ED, Allison TJ, Lee ND, Fisher $\mathrm{J}$ et al (2004) Immunophenotype of blood lymphocytes in neuroblastoma-associated opsoclonus-myoclonus. J Pediatr Hematol Oncol 26:718-723

18. Pranzatelli MR, Travelstead AL, Tate ED, Allison TJ, Moticka EJ, Franz DN et al (2004) B- and T-cell markers in opsoclonusmyoclonus syndrome. Neurology 62:1526-1532

19. Raffaghello L, Conte M, De Grandis E, Pistoia V (2009) Immunological mechanism inopsoclonus-myoclonus associated neuroblastoma. Eur J Paediatr Neurol 13:219-223

20. Sottini A, Micheli R, Ghidini C, Valotti M, Airo P, Caimi L et al (2007) T-lymphocyte production, function and death in children who recovered from opsoclonus-myoclonus syndrome. Pediatr Hematol Oncol 24:23-27

21. Tate ED, Alison TJ, Pranzatelli MR, Verhuldt SJ (2005) Neuroepidemiological trends in 105 US cases of pediatric opsoclonusmyoclonus syndrome. J Pediatr Oncol Nurs 22:8-19

22. Tsiodras S, Kelesidis I, Kelesidis T, Stamboulis E, Giamarellou H (2005) Central nervous system manifestations of Mycoplasma pneumoniae infections. J Infect 51:343-354

23. Verma A, Brozman B (2002) Opsoclonus-myoclonus syndrome following Epstein-Barr virus infection. Neurology 58:1131-1132

24. Waites KB, Talkington DF (2004) Mycoplasma pneumoniae and its role as a human pathogen. Clin Microbiol Rev 4:697-728

25. Waris ME, Toikka P, Saarinen T, Nikkari S, Meurman O, Vainionpää R et al (1998) Diagnosis of Mycoplasma pneumoniae in children. J Clin Microbiol 36:3155-3159

26. Wilson $J$ (2006) Neuroimmunology of dancing eye syndrome in children. Developmental Medicine Child Neurology 48:693-696

27. Wong A (2007) An update on opsoclonus. Curr Opin Neurol 20:25-31

28. Yiş U, Kurul SH, Cakmakçi H, Dirik E (2008) Mycoplasma pneumoniae: nervous system complications in childhood and review of the literature. Eur J Pediatr 167:973-978 